\title{
MODERN FYSICS PHALLACIES: THE BEST WAY NOT TO UNIFY PHYSICS
}

\author{
JAMES E. BEICHLER
}

\author{
Research Institute for Paraphysics, Retired \\ P.O. Box 624, Belpre, Ohio 45714 USA \\ Jebcolst@aol.com
}

\begin{abstract}
Too many physicists believe the 'phallacy' that the quantum is more fundamental than relativity without any valid supporting evidence, so the earliest attempts to unify physics based on the continuity of relativity have been all but abandoned. This belief is probably due to the wealth of pro-quantum propaganda and general 'phallacies in fysics' that were spread during the second quarter of the twentieth century, although serious 'phallacies' exist throughout physics on both sides of the debate. Yet both approaches are basically flawed because both relativity and the quantum theory are incomplete and grossly misunderstood as they now stand. Had either side of the quantum versus relativity controversy sought common ground between the two worldviews, total unification would have been accomplished long ago. The point is, literally, that the discrete quantum, continuous relativity, basic physical geometry, theoretical mathematics and classical physics all share one common characteristic that has never been fully explored or explained - a paradoxical duality between a dimensionless point (discrete) and an extended length (continuity) in any dimension - and if the problem of unification is approached from an understanding of how this paradox relates to each paradigm, all of physics and indeed all of science could be unified under a single new theoretical paradigm.
\end{abstract}

Keywords: unification, single field theory, unified field theory, quantized space-time, five-dimensional space-time, quantum, relativity, hidden variables, Einstein, Kaluza, Klein, Clifford

\section{Introduction}

It might seem as though the unification of physics within a single paradigmatic theory only became the primary goal of science toward the end of the twentieth century when scientists began talking about TOEs, but this would not be true. Complete unification was the original goal of Einstein and a few other leading physicists throughout the 1920s and into the $1960 \mathrm{~s}$, a time during which quantum theorists were attempting to iron out their own unique set of fundamental problems. The unification of physics under the guise of the quantum paradigm only emerged during the 1970 s and has since overshadowed all attempts to unify physics upon the fundamental principles of relativity, while general relativity has never really taken advantage of the advances in geometry that occurred in its early years and has theoretically stagnated in the ensuing decades.

The necessity to unify physics has recently passed beyond a philosophical issue and belief by some scientists to a desperate measure of practical significance in just the past few decades, but the basis upon which unification should proceed is still questionable. Unfortunately there has never been a method, either mathematical or physical, by which a three-dimensional space can be generated from two or more discrete dimensionless points. This geometrical shortcoming raises the question - how can the dimensionless point-particles of the Standard Model, which presently dominates physics, be extended to account for the three-dimensional space in which the physical interactions they describe occur? It also raises the question how a metric (symmetric) geometry of surfaces alone can be expected to completely explain gravity when it is only represented by a partial or incomplete Riemannian geometry.

General relativity and the Standard Model of particles seem to be and in many instances are physical opposites. They thus form a perfect duality as the modern paradigms of physics. This duality becomes apparent under many different guises, some of which are necessary, but others that are frivolous and still others that are completely irrelevant and misleading such as determinism and indeterminism, discrete and continuous, classical and modern. The only duality worthy of real consideration is function and form which act through the physical auspices of point- and extension-space geometries. For its own purposes, quantum theory tries as best it can to be non-geometric, but in the end it is nothing but geometry. Yet the quantum theory, as it is presently utilized, is also purely functional within the context of relativistic form (structure).

Fortunately, these questions can now be answered. The answer, however, does not favor the Standard Model of the quantum as it is presently interpreted. Nor does it favor the other great paradigm represented by general relativity, which is also incomplete in spite of all of its many observational and experimental successes. Instead, a unified field theory based on the continuity inherent in a newer extended theory of general relativity that completely unifies the quantum and relativity and completely incorporates the best features of the quantum and Standard Model as well as the Superstring, Brane and commonly accepted classical 
theories, free from all the prejudices and 'phallacies in modern fysics', is now called for and can be obtained.

\section{The search for unity}

The original unified field theories based on general relativity were never quite 'that' popular during their heyday between 1918 and 1960. They were overshadowed by the growth of nuclear physics and the quantum paradigm which had no need to unify with gravity (and electromagnetism) at the time. Even though the classical unification theories have now been all but abandoned, those first attempts are still valid if not for their vastly limited successes, and there were no real physical successes although there were mathematical and conceptual advances spawned by the attempts, but for their physical failures which indicated that an analysis of the mathematical advances yields clues to the present problems of all physical paradigms. Why did these attempts to unify the different paradigms fail when they used a more generalized versions of a Riemannian geometry that so successfully explained gravity were applied? They failed because the new generalized geometries that emerged were misunderstood and misrepresented by the physics with which they became associated.

Relativity based unification is usually represented by the various attempts of a small number of scientists to develop a single theory based on a unified field from which both gravity and electromagnetism would emerge as equal partners. Yet this particular interpretation of history is as much a 'phallacy of fysics' as it is commonly believed among members of the scientific community. Even if history is made more accurate by stating that after 1890 scientists began to think that matter and matter theory should be based upon electricity and electrical theory rather than gravity theory, so when Einstein demonstrated in 1915 that matter was best explained on the basis of a space-time curvature that was associated with gravity, the scientific community was taken by surprise and the 'phallacy' remained uncorrected. The 'phallacy' runs deeper and has been more harmful than ever suggested by the continuing successes of the original theory of general relativity.

The commonly accepted history of classical unification is a 'phallacy' that has doomed general relativity to remain a static theory since unifying electromagnetism and gravity seemed to be the only option considered for advancing relativity theory even though scientists and mathematicians determined that the Riemannian geometry used by Einstein to explain gravity alone was incomplete. In other words, the more general non-Riemannian geometries that were inspired by general relativity should have been used to extend gravity theory itself, independent of any attempts to unify gravity and electromagnetism, but they never were used properly and the 'phallacy' that general relativity offered a complete theory of gravity grew stronger over the years since. That 'phallacy' has been maintained as an incorruptible fact of science for nearly a century.

Under these circumstances, relativity theory surrendered the high ground of theoretical physics to the quantum paradigm and the quantum theory alone has led science down an ever narrowing path of progress as did Newtonianism in the last few decades of the nineteenth century. In the end, the first work on unification resulted more from the observation that Riemannian geometry as used by Einstein in general relativity was incomplete, so the unified field theories were more attempts to render relativity even more general by expanding the geometry and hoping that the expanded geometry would include electromagnetism which was impossible under the conditions. All classical unification theories, including Kaluza's five-dimensional theory, wrongly assumed curvature to be an intrinsic property of the space-time continuum based on the new nonRiemannian geometries.

\subsection{Intrinsic curvature models:}

In 1917, the mathematicians Gerhard Hessenberg [1] and Tullio Levi-Civita [2] were inspired by the immediate success of general relativity to expand and further generalize Riemannian geometry. The physicist Hermann Weyl [3] also began his unification in 1917 from a strictly mathematical expansion of Riemannian geometry to include (eventually) his concept of gauge in 1918 and 1919 [4]. However, his gauge theory of unification was susceptible to fundamental problems and he abandoned his attempts to unify physics, but not the mathematics of his gauge theory. Gauge theory subsequently found a home in quantum theory. Arthur Eddington is better known for his observational confirmation of light bending during an eclipse at Tenerife in 1919, but he followed Weyl and developed a unification theory based on an affine connection in 1921 [5]. In some ways these nonRiemannian geometries implied a higher-dimensional embedding manifold, but the geometries were instead limited to intrinsic points in the four-dimensional curvature of space-time continuum. But then you can justify anything in mathematics by how you define your parameters even if what you do is physically illogical.

The mathematician Élie Cartan developed an alternative form of non-Riemannian geometry in 1923 [6] and applied the geometry to the question if unification in 1924 [7]. This led to Einstein's attempt 
to base unification on the idea of parallel transport using Cartan's geometry in 1929 [8]. The EinsteinCartan theory employed an anti-symmetric tensor in addition to the normal symmetric tensor to represent curvature at a point in space-time. The antisymmetric tensor represented a 'twist' or torsion at points in space that was completely absent from the original Riemannian geometry used by Einstein. Some scientists still use the Einstein-Cartan model and have attempted to develop a new fifth fundamental force in nature called gravitational torsion [9], but their work has not been well received by the scientific community at large.

All of these men noticed that the tensors used to represent the metric curvature of space-time in general relativity were located at points of space, but only took account of the continuity of the curvature 'through the point' rather than any specific physical characteristics of space-time 'at the point'.

Einstein tensor in 3-D space

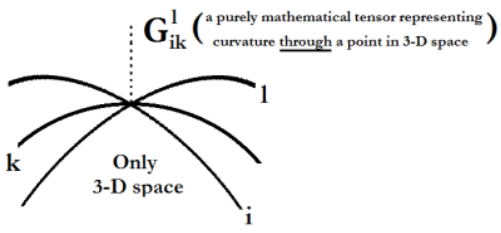

The new geometries that emerged were thus based on how the characteristics of the points themselves could be modified to generalize the Riemannian geometry used in general relativity. However, the new geometries seemed to be tied up within or limited to some form of internal geometry to the point without addressing (1) the issue of 'point to point' continuity within the Riemannian manifold and (2) without introducing any new dimensions to space-time. Pauli later referred to these geometries as 'tangent spaces' [10] because they only altered the geometry at the points in space-time tangent to the standard Riemannian curvature.

Yet even these geometries missed the point because they treated points and the metric curvature differently, i.e. they noted the geometrical problem of points, but did not relate their solutions to the continuity through the points that was already thought to have been (but was not completely) explained by the Riemannian metric.

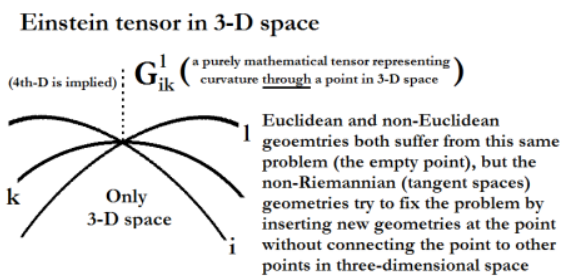

In other words, the point-to-point continuity assumed in normal geometry was not carried over to the geometries of the points themselves, so the points themselves remained independent and discrete from the four-dimensional curved space-time continuum of general relativity. All that connected the different geometries (Riemannian and non-Riemannian) were the individual unconnected points whereas connection should be through all points in the different spaces (manifold and embedded) consecutively and simultaneously. Yet the same was also true for the hyper-dimensional unification theories even though they assumed that curvature is an extrinsic property of the space-time continuum.

\subsection{Extrinsic curvature models:}

Kaluza sought to solve the same problem of unifying gravity and electromagnetism in 1921 [11] by assuming that the four-dimensional space-time continuum was embedded in a five-dimensional manifold. His theory enjoyed a limited albeit short success, but no one noticed that he made the same mistake as those who sought to solve the point problem intrinsically.

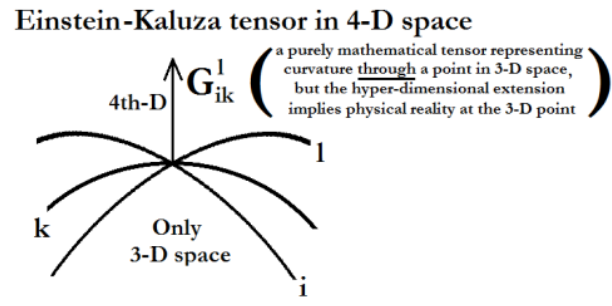

He did not tie the individual discrete points to each other in normal space-time let alone fourdimensionally across the fifth direction of space-time.

Each point in normal space-time was individually extended into the higher dimension and each individual discrete point was subject to the same mathematical conditions.

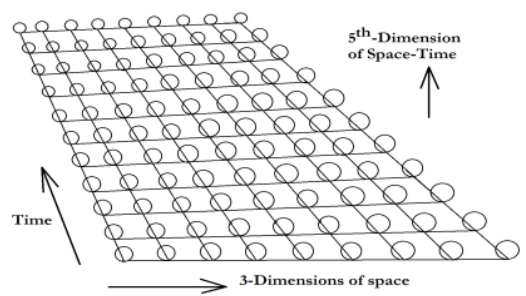

The linear extension into the higher embedding dimension was called an A-line. Each A-line looped around to itself so the higher dimension was closed with respect to each point and each A-line was the same length. These conditions, together called the cylindrical condition, were necessary for Kaluza to develop the mathematical model that he did, but the cylindrical condition also limited the fivedimensional model to only repeating the Maxwell electromagnetic equations at best without providing 
any new physics or predictions by which the theoretical model could be tested.

The cylindrical condition was sufficient to develop and support Kaluza's particular mathematical model, but it was merely sufficient without being necessary. So the cylindrical condition was also the source of the downfall of the theory because it over restricted the theory leading to an inherent incompleteness of the extended higherdimensional geometry.

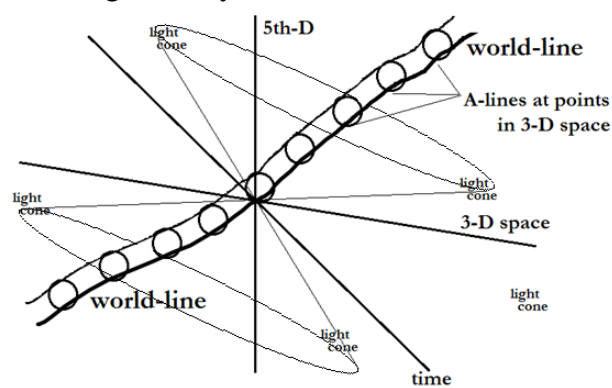

The world-line of an event in the light cone of 4-D space-time is an infinitesimally miniscule cylinder in the 5 th-D of space-time

Although it seemed a necessity at the time, the cylindrical condition merely implied that each point was connected to the next point providing continuity in four-dimensional space-time. Continuity was merely an illusion when the geometry was modeled along a space-time diagram and the three dimensions of normal space were reduced to one dimension on drawing paper. So Kaluza's model suffered from the same over-restriction and incompleteness from which the intrinsic non-Riemannian models suffered -the lack of demonstrating continuity between consecutive infinitesimal points.

Kaluza further suggested that the A-line loops must be extremely small because the higher dimension was beyond observation and even related that notion to the quantum. But it was not until five years later that Oskar Klein [12] published papers extending Kaluza's five-dimensional model to include the quantum. Klein noticed that the A-line loops formed a periodicity that could be quantized and thus the Kaluza-Klein model was born. Klein continued his theoretical research trying to quantize the Kaluza model over the next three decades [13], but each successive model proved to be a failure. Einstein and a few others also continued to develop the hyper-dimensional concept, but abandoned that attempt in the 1940s.

The hyper-dimensional approach perhaps seemed the most natural method to extend general relativity and include electromagnetism because Riemann had mandated that every $n$-dimensional space was embedded in an $n+1$-dimensional manifold. Although Kaluza was the first to try and expand Einstein's relativity using this method, W.K.
Clifford had tried to develop theories based on fourdimensional spaces as early as the 1870s and his work was influential during the late nineteenth and into the twentieth century.

The Evolution of Classical Unified Field Theories

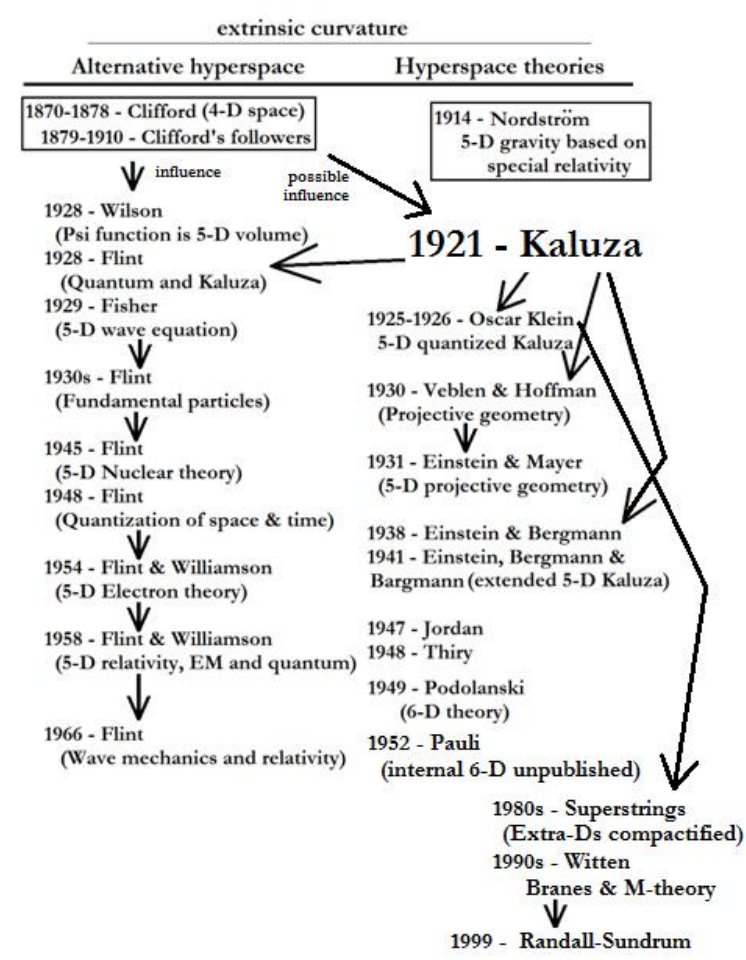

Together these two men's work was enough to overcome scientific prejudices against using higherdimensional spaces due to the simple fact that they cannot normally be sensed or detected. However they caused a philosophical backlash in the development of positivism, so their greatest influence would not come until the 1980s with the development of superstring theories.

\subsection{The final assault on classical unification}

After his hyperspatial interlude of the 1930s, Einstein returned to his 1925 [14] attempt to expand general relativity to include electromagnetism by adding a non-symmetric portion to his curvature tensor. The final thrust toward these unified field theories began in 1944. They were made by Frank R. Saxby [15], Erwin Schrödinger [16] and Einstein [17] who came upon the same model - the non-symmetric model independently from different theoretical approaches. Schrödinger came upon the model by combining the 1929 Einstein-Cartan anti-symmetric model with the earlier work done on affine geometries, while Einstein just added a non-symmetric portion to create a more generalized curvature tensor. Yet the resulting models were fundamentally equivalent, so the final Einstein-Schrödinger non-symmetric theory can be 
viewed as the culmination of all the previous intrinsic curvature theories.

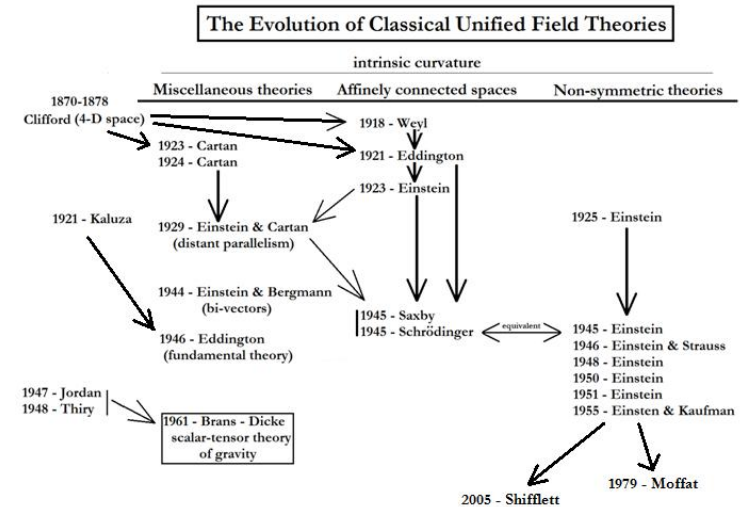

Einstein worked on this model until his death in 1955 [18], but the calculations from his non-symmetric model for charged particles always yielded values effect that were far too small to account for electromagnetism [19]. Einstein also obtained the same results for the motion of charged and noncharged particles, which implied a problem with his fundamental assumption that the non-symmetric portion of the tensor represented electromagnetism and not something else. These problems were never worked out and the non-symmetric model has been all but abandoned since shortly after Einstein's death.

\section{The fundamental problem}

There is a very important reason for the failures of these theories which is related to the calculated discrepancy between a moving particle in the nonsymmetric model and that of a real charged particle moving in space-time. This discrepancy is due to the fact that Einstein and the others working toward a unified field theory were working with an incomplete geometrical picture of space and manifolds that did not include both point-space and extension-space (metric-space) as connected geometric equals. The development of non-Riemannian geometries went only part way toward solving this problem as did the hyper-dimensional models, but they each went different parts of the way. Space theory did not need a new embedded geometry whether non-Riemannian or pseudo-Riemannian to represent the point itself. They instead needed to fully integrate the infinitesimal point into the old Riemannian geometry and find the consequences of doing so. The nonRiemannian geometries that were developed to support unification represented the wrong approach to the common problem when the problem actually called for super- or better yet hyper-Riemannian geometries.
In other words, the non-symmetric tensor has nothing to do with electromagnetism because it represents a purely secondary effect of gravity due to the dualistic nature of space itself. John Moffat came to a similar conclusion in 1979 [20], but he reached the same conclusion from a completely different direction. The only others who presently seem interested in the non-symmetric theories are historians associated with the Einstein Papers project [21] and a few other historians [22] as well as the American physicist James Shifflett [23], yet none of them has yet hit upon this common fundamental problem that dooms all such theories to ultimate failure.

The Einstein metric tensor of classical unified field theories should have two parts - symmetric and non-symmetric - which represent the dualistic nature of the space-time continuum itself. The symmetric portion of the tensor yields space-time curvature to explain Newtonian gravity while the non-symmetric portion which was thought (wrongly) by Einstein and others to explain electromagnetism actually predicts a secondary gravitational effect that is commonly (but wrongly) referred to as Dark Matter and Dark Energy. Dark Matter is not a separate or new form of matter and Dark Energy is not really energy, but field potential that becomes energy only when it interacts with normal matter. Only the scientists who have investigated the 'torsional gravity field' have correctly reached a similar conclusion, but even they have also interpreted the resulting effect wrongly and not related the concept 'torsion' or 'twist' inherent in the geometric points of space-time to Dark Matter and Dark Energy because they are still working with an outmoded intrinsically curved model of the spacetime continuum.

Calculations by Einstein and others were many magnitudes too small to account for the motion of charged particles because they represented a nonlocal gravitational effect rather the electromagnetic action. Einstein and others who attempted to apply the non-symmetric geometry to the motion of charged particles were not calculating an electromagnetic effect, but were instead calculating the motion due to the point-space geometry that can now be associated with Dark Matter and Dark Energy. This means that normal matter (baryonic) is the source of astronomical phenomena associated with Dark Matter and Dark Energy. Non-symmetry has nothing to do electromagnetism, but with instead deals only with the normal geometry of space as it is analyzed from the two different dualistic perspectives of point-space and extension-space. 


\subsection{Kaluza incomplete}

The five-dimensional Kaluza model also suffered a similar incompleteness. Although Kaluza was able to duplicate Maxwell's formulas (some thought even this duplication was artificial) using his model, the geometry he used (based on the cylindrical condition) was too restrictive and did not allow any further deductions or testable predictions about either the nature of the higher dimension of space or normal phenomena in our four-dimensional space-time. Einstein and Peter G. Bergmann [24] tried to solve this problem in 1938 by rethinking Kaluza's fivedimensional model without the cylindrical condition.

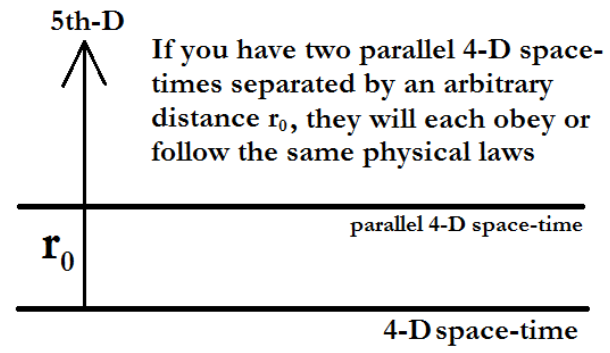

They proved that if the cylindrical condition is modified, the embedding manifold itself could be closed as a whole rather than piecemeal in a point-bypoint fashion as in Kaluza's original model. This implied that the higher dimension could consist of an infinite number of parallel four-dimensional spacetimes, constituting a specific 'thickness' of such submanifolds, without changing the physics of normal four-dimensional space-time. So only what is done along the fifth dimension of space could affect or influence the physics of normal four-dimensional space-time.

Yet they mistakenly stopped with this proof and did not attempt to develop any picture, properties or rules regarding the geometry of the extra dimension of space. They did not realize that the higher embedding dimension or manifold must be geometrically different from the normal three dimensions of space because it has never been detected or observed and matter is only three dimensional, at least outwardly relative to other bits of matter.

Going even further it is easy to see that both the non-symmetric and the five-dimensional models of space-time are incomplete in themselves yet complement each other because they are based on the simple geometrical 'phallacy' that space can only be modeled by a simple metric or extension-space geometry even though space really has two distinct elements or parts - point and extension. Therefore, every physical model of reality is incomplete according to the simple Euclidean and Riemannian geometries used in normal physics and the standard structure of space and time. Simple electromagnetism already has two parts - scalar and vector potential which represent the inherent extension- and pointspace geometries. In other words, electromagnetic theory supports the simple notion that the normal geometry of space has two parts, so gravity must act and react in the same way to the same shared space. Any gravity theory or model of space must also account for both of these fundamental aspects of real physical space, so gravity must also have two parts scalar and vector potential - to be completely represented in the geometry of relativity.

This is the same point that the original mathematicians and scientists working to unify relativity and electromagnetism indirectly but unsuccessfully tried to make. They failed in their attempts because they either did not go far enough or they were sidetracked into theoretical dead ends and the geometric message they hoped to implement in physics got lost in the ensuing decades. All of the geometries used in unification are incomplete for the same reason, they do not take into account the natural duality of space and no one seems to ever have noticed this discrepancy. To complicate the problem further, with Einstein dead (1955) and no one else alive to carry on and complete his vision of a unified field theory, unification based on general relativity and the continuity of the space-time continuum all but died away while the quantum theory and relativity continued to follow their own individual evolutionary courses under the assumption that they are completely and irrevocably incompatible, which is another of the great 'phallacies of fysics'.

\subsection{Compatibility implied but still denied}

During the 1960s and 1970s, a number of theoretical advances (QED, QFT, QCD, the electroweak force and so on) in quantum theory convinced quantum theorists and the scientific community as a whole that unification was a good thing, but this new form of unification was based on the quantum model and sought to overthrow or replace general relativity with a whole new physical model. However, the quantum theory will never overthrow relativity and relativity will never replace the quantum because they are fundamentally different approaches to understanding nature and material reality. In other words, they form a necessary duality in nature. Relativity seems to be all about form (structure) and the quantum seems to be all about function, which come together as one of the most fundamental dualities (known as noncommuting quantities in physics) in nature, but there is always a bit of each in the other.

This situation perfectly mimics the philosophical concept that is normally expressed in Eastern philosophy as yin and yang and even though 
they are opposites in a sense, one cannot exist without the other.

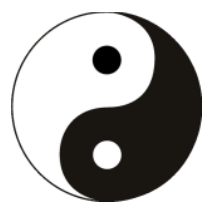

The structure or form of relativity provides the context against which the action or function of the quantum makes sense, while the function of the quantum alters the structural landscape or form provided by relativity. So at some more fundamental level than their apparent duality, relativity and quantum become each other. The same fundamental concepts appear in western philosophy as thesis and antithesis, which are normally resolved through synthesis rather than conflict, so all of the conflict between relativity and the quantum is nothing but a grand 'phallacy' of misinterpretation.

\section{Incomplete quantum theories}

Be that as it may, there are other reasons that the quantum and relativity can be neither unified nor overcome by the other at this point in their evolution. The individual quantum attempts at unification (the so-called TOEs) are also incomplete within the context of their own basic and fundamental precepts without any reference to the geometrical problems of the classical attempts at unification. In fact, they fall prey to the same problem of failing to distinguish between point-space and extension-space that ultimately doomed the attempts to base a unified field theory on general relativity.

The standard model of particles is by far the most popular of the modern quantum unification attempts. In this model, quantum theorists claim particles are discrete points in fields (QFT) rather than extended bodies because theoreticians cannot deal with the concept of continuity - yet the quantum fields that they postulate to accompany pointparticles are essentially continuous. Points are 'nothing', literally infinitesimal non-dimensional elements of reality, so they cannot be particles that are 'something', while particle theorists still cannot explain how to go from their discrete point-particles to observed extended particles without inventing unnecessary new particles, ad infinitum.

The Superstring model has perhaps become the next most popular theoretical quantum model of reality over the past few decades and some believe that the standard model and the superstring model will eventually merge together, but two wrongs do not make a right. Superstrings are not immune to the same difficulties that other theories fail upon.

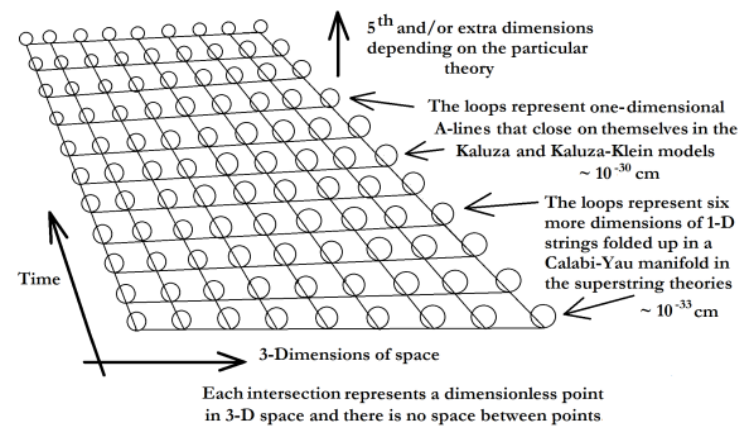

The superstring model is based loosely on the Kaluza-Klein model of the 1920s except for the fact that superstring theorists need ten, eleven, twenty-six or more dimensions to explain the characteristics of particles instead of the original five Kaluza used. Each point in four-dimensional space-time is uniquely extended into itself to create a separate sixdimensional Calabi-Yau manifold of onedimensional strings whose individual vibrations and combinations of their vibrational states give material particles their unique physical characteristics. The physics implied by this model seems to emerge out of the nothingness (non-substantiality) of the theoretical mathematical model, which is itself problematical.

Yet even without making any criticisms of the superstring theory itself upon its own merits, it is easy to see that this model carries with it the same problems of connectivity of contiguous points in three-dimensional space and one-dimensional time that plagued the Kaluza model upon which it is ultimately based. So the superstring models are themselves incomplete because the Calabi-Yau sixdimensional manifolds are no more than tangent geometries of the same type as the non-Riemannian geometries upon which the failed unified field theories were based, except for the fact that they are more complex internally due to their hyperdimensional nature.

The Randall-Sundrum [25] and similar brane models that have emerged from the fold of the superstring models break all the rules and throw out the cylindrical condition without replacing it with any equivalent mathematical rules by which to develop their physical model. They do so by positing two different four-dimensional space-time 'branes' or membranes that are swimming within an undefined five-dimensional bulk. The branes can be any distance apart, either great or small, so the concept of closure in the embedding manifold (or in this case the bulk) has been completely abandoned. Yet the cylindrical condition of point-by-point closure was mathematically necessary in the Kaluza model upon which the superstring and brane theories were originally based, so throwing out those mathematical 
conditions should negate the internal mathematical logical consistency of such brane models.

Furthermore, this configuration does nothing to solve or even identify the point- and extension-space problem within the four-dimensional branes that it posits, so problems with this model abound. Yet the model is not without some merit and can easily be corrected to bring it back to Kaluza-style closure even if the point/extension problem is not fixed.

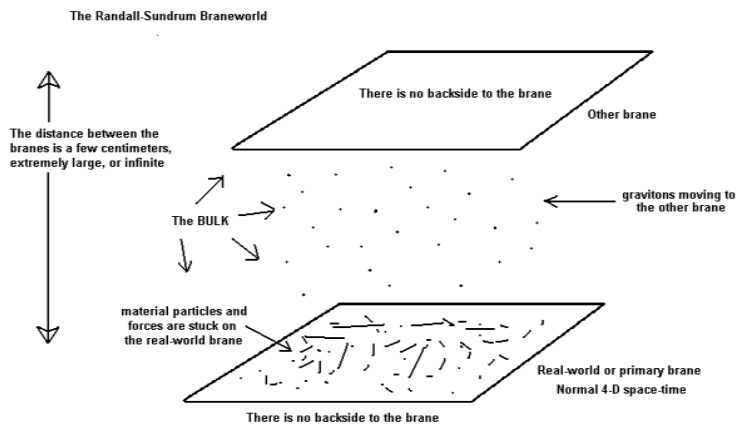

This model has on open geometrical structure whereas the geometry upon which it is based was closed with respect to the embedding dimension. Yet if the distant or infinitely placed secondary brane is just rotated into a position where it becomes the underside of the original primary brane, a model worthy of the 1938 Einstein-Bergmann proof emerges. A new form of unification is thus implied if the point/extension problem can be overcome.

Quantum theory as a whole obviously suffers from the same point/extension duality as the classical unified field theories, while the concept of a pointparticle is itself a serious 'phallacy'. The very suggestion of point-particles should raise the fundamental question 'What distinguishes pointparticles from other geometric points in the so-called quantum fields associated with the point-particles?' Yet this and similar questions are neither asked nor answered in the Standard Model. It is ignored, which reduces QED, QCD and the Standard Model to nothing more nor less than useful, sophisticated, extremely accurate, but very complex physical 'approximation of reality' methods that do not really represent physical reality as a true theory should. All quantum field theories fit this same mold.

Even theoretical mathematics suffers from the same point/extension problem that physical theories fail upon. In 1900 the mathematician David Hilbert [26] listed the seven major unsolved problems facing mathematics. The first and foremost of these, the continuity problem, is essentially the same as the point/extension problem in physics only it is couched in mathematical terminology. And like physics, this problem has yet to be solved with any degree of certainty in mathematics. Yet nature works and progresses on in spite of these human problems of interpretation, while the relativity and quantum paradigms have reached extremely accurate if not somewhat troubled degrees of accuracy in describing the inner workings of nature.

Given that all present paradigms of physics and mathematics suffer from this same apparent schizophrenic dichotomy of nature, the only possible answer to unification is to merge or blend the various theories together as they are without making major changes (except interpretative) in them - in other words save the good and get rid of the bad in each applicable theory and paradigm. Science must render each paradigm as internally complete as possible, which means solving the point/extension problem as it relates to each particular paradigm and theory, then finding the point where they are compatible by developing a proper geometry that includes both the point and extension (metric) as equal partners in our geometric reality. Doing so will merge the seemingly incompatible paradigms into one. This procedure has become necessary since all present physical theories of reality suffer from the same problem by failing to adequately account for how an infinite number of infinitesimal points can yield a continuous extended space-time. This problem is just more fundamental and immediate in theoretical physics where nature makes the rules than in mathematics where the mathematician makes the rules, so it has evolved more rapidly and profoundly into a crisis for physicists to solve.

\section{The classical point of compatibility}

The physical problem of unification is directly related to the mathematical problem of infinitesimals and continuity which has as long a history as the physical problem of points and extent in space and time because they are two versions of the same problem. With regard to the mathematical concepts, Weyl [27] still took an oversimplified view of the problem in 1927 and compared the solution to Galileo's "bending theory". He stated that "If a curve consists of infinitely many straight 'line elements', then a tangent can simply be conceived as indicating the direction of the individual line segment; it joins two 'consecutive' points on the curve." Yet this is the same problem faced many centuries earlier by Zeno, Euclid, Archimedes, Nicole Oresme and Isaac Newton [28].

Weyl and others had determined the central fundamental problem in geometry - infinitesimals, but had yet to think of a truly original solution to overcome the problem. Yet scientists and mathematicians have wrongly acted as if the problem of infinitesimals automatically turning into continuities had been completely solved for too long 
even though they had known that it has not been solved and thus missed the real solution to the compatibility problem in physics. The solution is simple. Theoreticians have to reverse their logic and ask how to go from infinitesimal points to extended continuities rather than from continuous extensions to infinitesimal points as a limit. The success of general relativity and subsequent search for a unified field theory only exacerbated this problem.

As far as science is concerned there are two and only two instances that demonstrate the physical reality of infinitesimal dimensionless points - centers of rotation and mass/gravity. These two examples imply the necessity of a single dual point- and extension-space geometry in physics to explain material reality instead of the metric geometries normally used in classical gravity theory. In the case of Newtonian gravity, only a center of mass or center of gravity is needed for point-to-point calculations of gravitational forces between material bodies which ignores the concept of a center of rotation even though gravitationally derived orbits have centers of rotation.

Maxwellian electromagnetic theory does not suffer from this particular problem, but Oliver Heaviside noted the differences between the two existing concepts of natural forces and wrote a new gravity equation in 1891 [29] that directly expresses gravity in terms of both a point- and extension-space geometry.

$$
\mathbf{F}=\mathbf{m g}+\mathbf{p} \times \mathbf{S}
$$

Heaviside interpreted the new term for gravity as a true centrifugal force on orbiting bodies, which is exactly what it is if the vector $\mathbf{S}$ represents the total mass of the rest of the universe as opposed to the central body's field around which the body ' $\mathrm{m}$ ' orbits. In modern physics that total mass is represented by the overall Riemannian positive curvature of the universe.

Modern scientists have mistakenly interpreted this term as a new gravito-magnetic force, but have gotten it wrong and thus failed to develop experiments that can successfully test for this 'new force'. Heaviside only wrote his equation in order to render Maxwell's and Newton's theories symmetric to each other without lending the second gravity term any specific geometrical meaning beyond the effect of a centrifugal force. So it seems that gravity does have a real centrifugal component for objects orbiting other centers of gravity as Newton originally argued, while magnetism also acts centrifugally in the sense that the term ' $q \mathbf{v}$ cross $\mathbf{B}$ ' in the Lorentz equation represents the net external magnetic field through which the charge q travels, which is also true. So centrifugal forces only occur in with respect to natural forces and not when an object is centripetally moving around a center to which it materially attached.

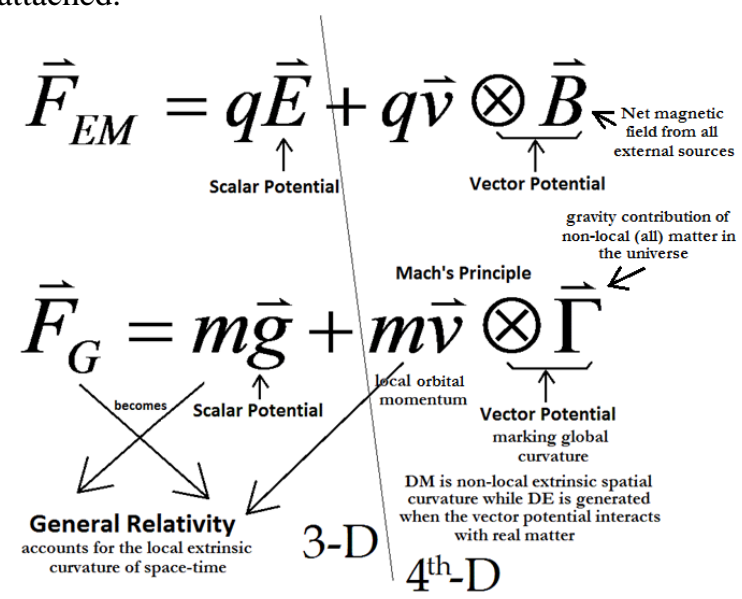

Heaviside's equation can be rewritten using the Greek letter Gamma to represent the rest of the matter in the universe that contributes 'equally and oppositely' to this gravitational centrifugal force. Perhaps this new term for gravity could be thought of as the mathematical representation of Mach's Principle, which it essentially is.

If other scientists had interpreted the equation correctly, they would have found that their 'new force' is associated with and can explain Dark Matter and Dark Energy within a Newtonian context.

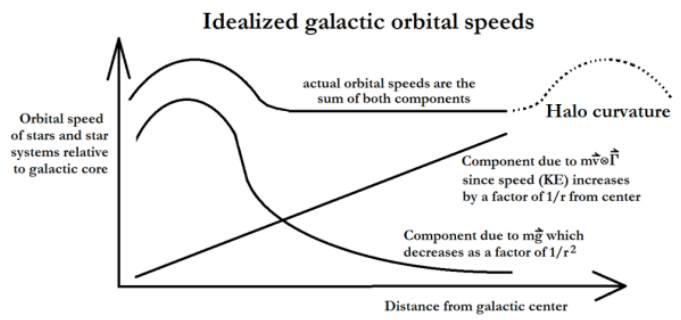

The halo is like a shadow or mirror image of the core curvature due to the centrifugal force (equal and opposite) of gravity due to the rest of the universe represented by $\Gamma$

The 'equal and opposite' force in this classical equation balances the gravity of the galactic core in such a way that it forms the curvature for the galactic halo without the presence of local matter. Yet no one has yet tried to relate the new gravitational term (gravnetism) to a dualistic geometry where normal gravity is supplied by the extension-space geometry and the new term is due to the point-space geometry, thus offering a new complete classical theory of gravity. This interpretation further implies that the curvature is a real part of an extrinsic geometry that requires the reality of a higher embedding dimension as Clifford suggested in 1870.

Einstein and Bergmann indirectly tried to solve the geometrical problem of points after their own manner in 1938, although that was not their purpose. So they stopped short of the answer because they only wanted to get rid of Kaluza's cylindrical 
condition to render the fifth dimension more realistic. They developed a geometric proof demonstrating the feasibility of the same large or macroscopic extra dimension of space, but gave no clues regarding the actual geometry of that fifth embedding dimension of the space-time continuum because they also missed the 'point'. Unfortunately, they also threw out Klein's extension of Kaluza's model to include the quantum when they threw out the Kaluza's cylindrical condition as it was originally foreseen. Klein's extension was incomplete since Kaluza's model was incomplete, which means that the superstring and brane theories are also incomplete. Every one of these theories misses the 'point' while hinting that the point has some special significance in physics.

Yet Klein had the right idea - quantize the extra dimension of space which automatically quantizes four-dimensional space-time. This was demonstrated indirectly by Einstein and Bergmann's 1938 mathematical proof. Neither Klein nor anyone else since 1900 has realized that Planck's constant is really the binding constant that ties space and time together, literally that which holds them together to form the space-time continuum marked by a fundamental standard 'unit of change'. This interpretation is completely compatible with the Heisenberg uncertainty principle and can be easily demonstrated as such [30]. The uncertainty principle hides or more accurately suppresses the concept of time in the relationship $\Delta \mathrm{x} \Delta \mathrm{p} \geq \mathrm{h} / 4 \pi$ and space in the relationship $\Delta \mathrm{E} \Delta \mathrm{t} \geq \mathrm{h} / 4 \pi$. Splitting time and space apart in the manner of the uncertainty principle necessarily invokes Planck's constant and the uncertainty associated with its application.

\section{Theorem of physical reality}

Given all of these failures of modern physics and the 'phallacies' that spawned them, the main question becomes how to get from the mathematical concept of dimensionless points - an infinite number of which make up an extended line of any length - to a physically real and continuously extended line even though two or more points put together by contact still reduce to a single point because they are dimensionless [31]. Solving this problem leads to a new mathematical theorem of physical reality. It is based on Gödel's theorem and a working definition of contiguous discrete dimensionless points.

Two or more infinitesimal points cannot be placed together in such a way that they form a continuous extended line even though a continuous extended line can be shortened until it reaches the limit of a single infinitesimal point. This statement constitutes a previously unrecognized fundamental paradox in science and mathematics. The points cannot be added together to form a line or surface because being dimensionless they would be superimposed one atop the other when they are placed 'next to' each other and could thus never form an extended line or surface. That is exactly the concept that Weyl was referring to and it is the 'crux' of the paradox between infinite and infinitesimal as well as infinitesimal and continuous. Newton solved the problem by the action of 'flow' or changes through points in his calculus of 'fluxions', which yielded only a partial and temporary fix to the problem. The later mathematical solution to this problem, defining an instantaneous velocity as the average speed as the time in the denominator goes to the limit of zero, was itself a formal recognition of the central point-space problem, but still only a partial and temporary fix of the problem. In other words, the mathematical problem can only be resolved by reference to another non-related system, i.e. physics or physical geometry, by applying Gödel's theorem an embedding the geometry in question within a higher-dimensional manifold.

Once the extrinsic higher dimension is invoked according to Gödel's theorem, two contiguous points can be defined as being so close to one another that another dimensionless point cannot be placed between them. The problem then disappears and an extended line or continuous surface can be formed. The points $\mathrm{A} \& \mathrm{~B}$ are contiguous because they are so close to each other that no other dimensionless point can be placed between them.

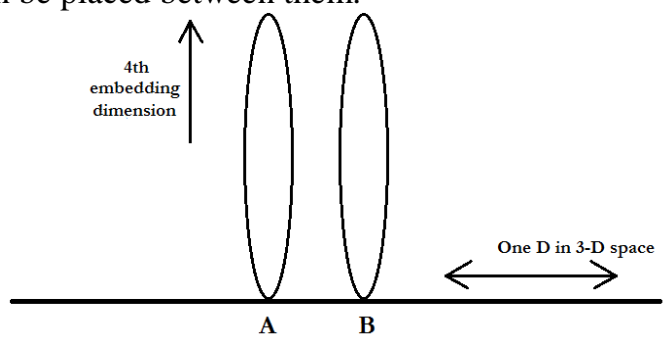

But we still cannot prove a continuous extension unless we curve the one-dimensional line in a second dimension, which yields a Riemannian twodimensional curved surface embedded in a higherdimensional manifold. This notion was implied by Riemann, but never stated in his development of a generalized differential geometry.

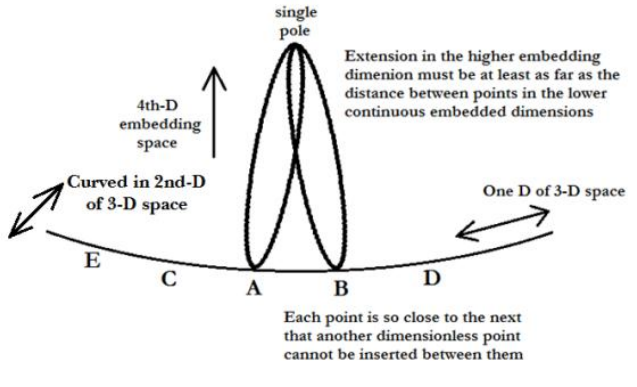


This structure yields a double-polar Riemannian three-dimensional space with the same physical characteristics - such as symmetries, translational and rotational motions - as our commonly experienced three-dimensional space embedded in a single-polar Riemannian manifold.

In total there are six different conditions upon which this geometrical structure is based. The first two are just the Kaluza (relaxed cylindrical) conditions: (1) All A-lines are closed with respect to their origin points, and (2) all A-lines must be of equal length. The next two conditions can be called the Clifford conditions because they were implied in W.K. Clifford's work in the 1870s [32]: (3) The onedimensional A-lines must all be at least as long as a circumference line that completely encircles the embedded three-dimensional space, and (4) all Alines must pass through or come together at a single common point: This point is the pole of a single-polar Riemannian sphere.

Two more conditions can be added to these: The Einstein condition [33] whereby (5) any theory must explain why we do not observe the higher dimension(s) and a general logical condition (6) that any change that influences the fifth direction, such as quantization, must affect the other dimensions of space which are a subset of the higher embedding dimension. There is also a General Rule of Unification that states since there are two parts point and extension - to our commonly experienced three-dimensional space, classical or Newtonian gravity as well as Einstein's gravity theory must also be affected by this duality as are electromagnetism and the quantum.

The Einstein condition is actually easy to explain since we do in fact sense the higher dimension of space. Superstring theories attempt to overcome this problem by compactifying the higher dimensions that they hypothesize to sizes too small to possibly detect, but that mathematical trick over restricts the geometry much as Kaluza's geometry was over restricted. On the other hand, there is no need to assume that we do not sense the higher dimension. Our normal senses act through the nervous system to neural nets and the brain. That is where our logical worldview emerges in mind and consciousness. However, we also have an intuitive sense of the world that goes beyond logic. Our intuitive sense comes from our geometrical connections to the rest of the universe through the higher dimension of space which acts through consciousness to mind. This is normally what people call our sixth sense, which is another story to be told elsewhere. [34]

\section{Real particle physics}

Under the geometrical and other conditions established by the physical reality theorem, material particles cannot exist until they fulfill the geometrical requirements of the higher-dimensional embedding manifold, i.e. real material particles can only have half-spin due to the Möbius like twist as the A-lines or a bundle of A-lines pass through the single pole.

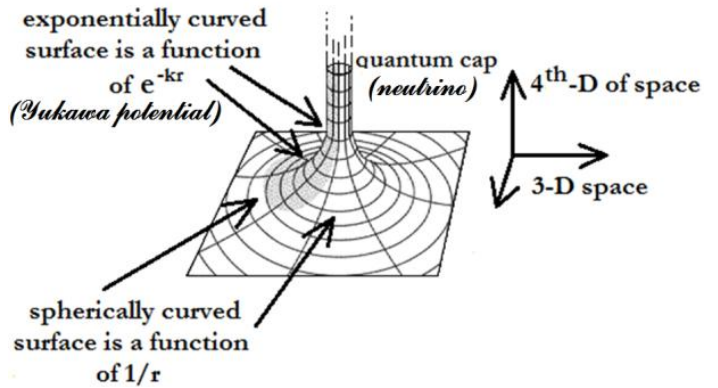

The real curvature in the higher dimension of spacetime that constitutes a particle never reaches the level of a singularity at its center since a quantum cap of 'sheet' curvature forms to prevent the singularity. A true mathematical singularity could never form in real space-time because the higher embedding dimension is closed, except for the possibility of the Big Bang singularity which predates the formation of the dimensions themselves.

Under these physical conditions, the number of real fundamental particles reduces to three - the proton, electron and neutrino - while the neutron would be a composite particle made from a proton and electron with the appropriate quantum cap or caps. There would be three different neutrinos corresponding to the quantum caps of the neutron, proton and electron while a fourth would correspond to the maximum curvature in the four-dimensional space-time continuum before an electron is formed. These three neutrinos would have the minimum amount of curvature to qualify as curvature of the 'sheet', which would yield particles whose threedimensional width would just equal the 'effective width' of the curved three-dimensional 'sheet' in the embedding dimension of space. The electron is the maximum amount of curvature that can occur before the 'sheet' folds on itself and creates the proton. Muons and tauons are just energetic electrons that have reached a state of quantum equilibrium, or rather the $n=2$ and $n=3$ principle quantum states of the electron.

Our normal four-dimensional space-time would form a 'sheet' of sorts orthogonal to the fifth direction, with other 'sheets' stacked one after the other to form the necessary continuity in the fifth direction. Each 'sheet' would have an effective width in the higher direction which would preserve Klein's contribution of quantizing the fifth direction to 
quantize the normal three-dimensions of space and one-dimension of time.

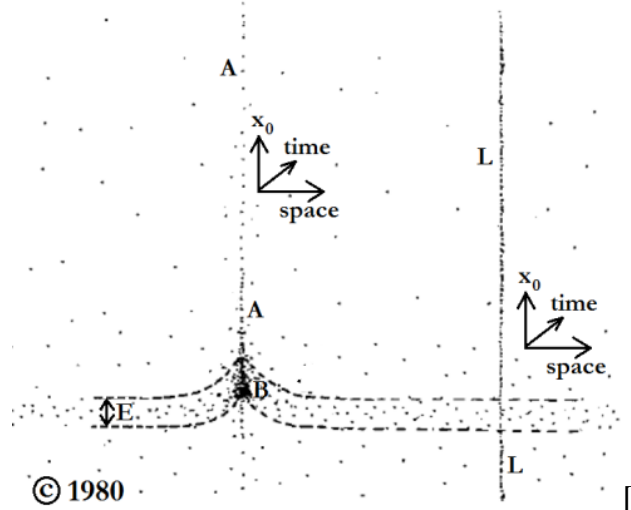

Each successive 'sheet', like stacked pages in a book, would correspond to a quantum number with the primary 'sheet' representing our commonly experienced space-time having a principle quantum number of one. The fifth direction would be characterized by a density variation of the single field over which the quantum numbered 'sheets' are superimposed. The primary 'sheet' that curves to form our world of matter, gravity and electromagnetism is just the densest part of the single field along the fifth direction of the space-time continuum. The single field density would decrease exponentially as the distance from the primary 'sheet' increases.

Real material particles would thus appear spherical in the three dimensions of space, but have the shape of an exponential curve in the higher spatial dimension. The gravitational force would amount to a 'surface tension' across the top of the 'sheet' and the electrical force of charged particles would reduce to a stress inside the particle, both emanating from the internal stress of particles being stretched in the fifth direction of space due to expansion.

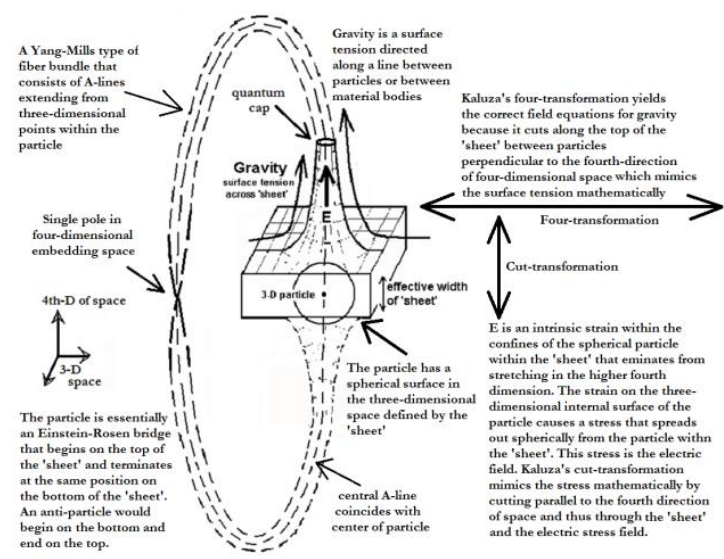

This model explains exactly why Kaluza's mathematical concepts of a 'four-transformation' and a 'cut-transformation' yielded the gravitational and electrical forces in three-dimensional space surrounding material particles. The 'fourtransformation' is the mathematical equivalent of the physical tug across the surface of the 'sheet' between particles, which is why gravity is always attractive, and the 'cut-transformation' literally cuts across the 'effective width' of the three-dimensional 'sheets' extended in the fifth direction of space-time to pick out the strain in the surrounding space at any point due to the internal particle 'stress' from being constantly stretched in the fifth direction of spacetime. Anti-particles would merely be equivalent curvatures along the underside of the 'sheet'.

\section{Atom and nucleus}

The atomic nucleus is easier to picture. Protons and neutrons would merely stack one on top of the other in the fifth direction of space to form the atomic nucleus. They would have to abide by boundary conditions at both the quantum cap, which would have the same 'effective width' as the primary 'sheet', and where the various nucleons come into contact with the 'sheet' that constitutes normal free space outside of the nucleus.

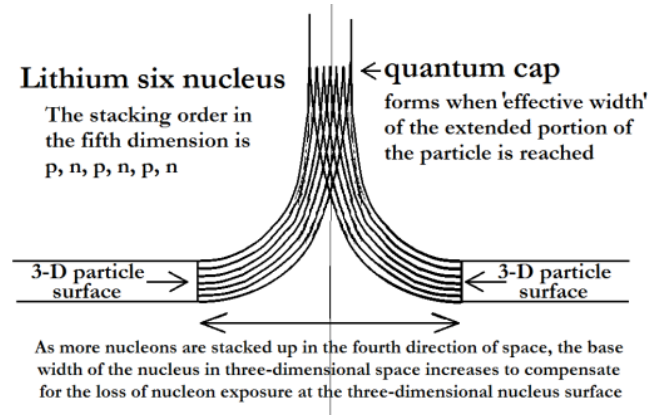

This configuration merges the shell and fluid drop models of nucleus into a single model which was previously thought impossible because these two successful models were thought to be (another phallacy) incompatible. The nucleus would exhibit 'shell' characteristics along the fifth direction of space-time according to the stacking order of the various nucleons. However, the nucleus would remain spherical with respect to its outer threedimensional surface. The stacking order within the 'effective width' of the primary 'sheet' would appear doubly rotated to an outside observer in threedimensional space which would allow each nucleon an equal presence on the outer three-dimensional surface of the nucleus as well as give the nucleons a certain fluidic appearance.

The Yukawa potential would reduce to the geometrical fitting or sliding 'force' between the contact surfaces of consecutive particles along their four-dimensional surfaces. $\mathrm{Vu} \mathrm{B}$. Ho [36] derived the Yukawa potential from general relativity in 2005 
although Henry T. Flint [37] had already incorporated it into his unified field model even though they were both working with the incomplete Einstein model of gravity. On the other hand, the electroweak force reduces to a type of point-to-point static electrical force between the curved contact surfaces within the nucleus. These would correspond to the forces connected to the individual geometrical points where the theoretical A-lines pass through the surfaces of successively stacked particles and continue on toward the single pole in the higher dimension. This model of the nuclear forces successfully reduces the nuclear forces to interior particulate aspects of electromagnetism and gravitation, which means that only two natural forces really exist in our world.

With the nucleus adequately modeled, the outside portion of the atom comes next. Although the curvature of the continuum inside the nucleus is extremely steep and exponentially shaped, the curvature outside of the nucleus is not nearly as radical.

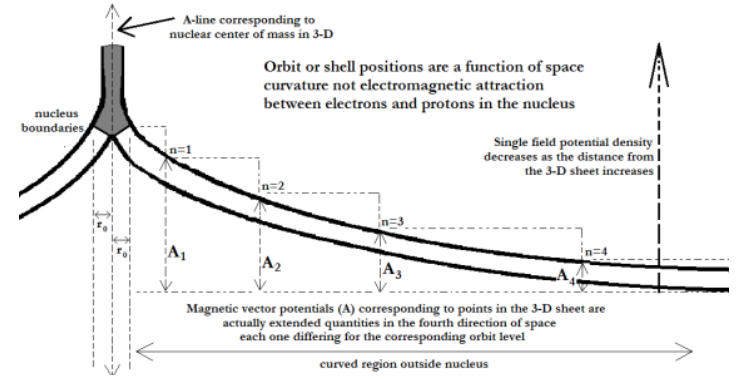

The curvature external to the nucleus is in fact quite gentle, but it cannot be ignored or dismissed as is the common practice in quantum mechanics. Each time the curvature drops along the fifth direction of spacetime by one effective width outside of the atom, a possible electron orbital state is distinguished. Electron orbits, both possible and filled, are determined by the quantized curvature of space rather than any purely quantum or electromagnetic restrictions.

When the curvature has become so slight that no such drop can occur, the outer physical limits of the atom have been reached. The drop distance in the fifth direction that marks successive electron shells or allowed orbits constitutes a specific magnetic vector potential equivalent, so when incoming light waves characterized by a specific magnetic vector potential that matches the equivalent drop potential the electromagnetic wave can be absorbed. This model thus accounts for both the emission and absorption spectra of atoms. The model also conforms to the notion that the first quantum orbit corresponds to a single DeBroglie wavelength of the orbiting electron, the second quantum orbit to two DeBroglie wavelengths of the electron and so on.
An extrinsic curvature within an embedding fifth dimension of the space-time continuum can thus account for the structure of the whole atom as summarized below. The quantum mechanical picture of a different physics for the interior of the nucleus and the external electron orbits disappears and is replaced by differently characterized forms of curvature due to the gravity and electromagnetic fields inside and outside the nucleus. These curvatures remain continuous at the outer wall of the nucleus providing boundary conditions for calculations. The potential characterized by geometrical points in free-space account for what is mistakenly called Dark Energy, while the same points that appear underneath the curvature that we normally associate with the interior of extended particles constitute the inertia of a particle. Both inertia and Dark Energy (really dark potential rather than realized or manifested energy) are point properties of space. The curvature itself, or the metric of general relativity, constitutes the gravitational mass of particles.

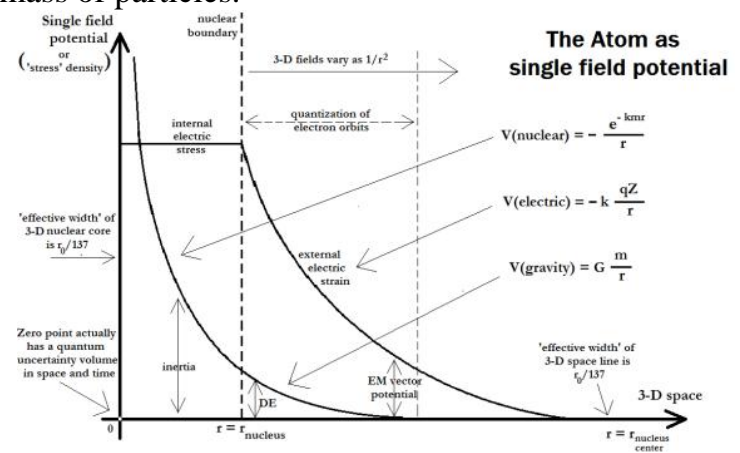

This means that the inertial and gravitational masses must be equal because the points under the curve constituting inertial points conform exactly to the metric curve that constitutes gravitational mass. This last statement represents a new and precise 'equivalence principle' that, unlike Einstein's original statement of the equivalence principle, is independent of accelerations and dynamic processes of change.

Furthermore, the energy-equivalence of the mass that is normally represented by Einstein's famous $\mathrm{E}=\mathrm{mc}^{2}$ formula is nothing more than the Dark Energy-points equivalent of the inertial points under the internal curvature that constitutes material particles. Inertia is more-or-less and for all intents and purposes Dark Energy that is confined to the interior of material particles by the quantized curvature of the continuum. This characterization makes more sense of the concept of a Higgs boson (which does not really exist as a real particle) and the quantized interaction between real particles and their corresponding quantum fields (the quantized curvature of space-time) than the original explanation 
by Higgs and others. In reality, particles are not singular central points within quantum fields, but extended particles within a quantized single field. All of the quantum fields together are no more than aspects of the single field more-or-less in the sense of David Bohm's concept of a single quantum potential field. No bosons are exchanged that can give pointparticles mass, but rather points in space are absorbed by moving extended particles as they transit relative space much as a ripple across the surface of a pool of water.

\section{Quantized curvature reaps fallout}

However, relating this explanation to the Higgs explanation of inertia implies the necessity to consider other issues regarding the relationship between the Standard Model of particles and this model of quantized curvature. The Standard Model perpetuates what is probably the greatest 'phallacy in fysics' and in all of science - the reality of pointparticles. Particles cannot be points simply because points have no size (zero extension by any other standard) which renders them dimensionless and that simple fact carries with it all of the problems and paradoxes that have plagued science and mathematics for nearly three millennia. In reality, all of the so called point-particles hypothesized by the Standard Model do not exist.

Only those extended particles with half-spin fulfill the new geometrical requirements for physical reality. All other so-called particles with spins other than one-half are merely temporary field pattern resonances that decay into real particles and/or photons. At best they can be called pseudo-particles (or wannabe particles) since they are intermediate physical responses to dynamic interactions with the curvature of the 'sheet' that lead to real particle creation. Their rapid decay is just their physical response to the inability to conform to geometrical and quantum requirements for physical reality.

All points in the four-dimensional space-time continuum that constitutes our material universe (in the 'sheet') are theoretically connected with all other points in the universe when they, or rather the A-lines and A-line bundles that distinguish their hyperdimensional existence, pass through the single pole in the higher embedding dimension. This connectivity is the primary source of the phenomenon called quantum entanglement. However, there are really two types of quantum entanglement, one along the fifth direction of the space-time continuum (rotational) and the other across or through the four-dimensional space-time 'sheet' (linear).

The higher level rotational entanglement that works along the fifth direction of the continuum (spin and polarization) can be instantaneous since no distance is traveled with normal space-time where the speed of light restricts signal transmission. The other lower level form of linear entanglement is purely geometrical based on a connection resulting from the continuity characterizing normal space-time. This form of entanglement is just the simplest type of Machian relativity based on Mach's Principle. This second type of entanglement was just that explained by Einstein, Boris Podolsky and Nathan Rosen in 1935 [38].

The dual geometric nature of space and entanglement also hint at solutions to a few other problems that have haunted classical and modern physics. Toward the end of the nineteenth century, some physicists pointed out that Maxwell's theory should include both longitudinal and transverse electromagnetic waves [39], but these waves were never detected. These waves can now be identified within this model. As a transverse wave spreads out spherically from its source in four-dimensional space-time a fifth component spreads out longitudinally along the fifth direction of the overall space-time structure. The notion of a magnetic vector potential is an essential component of Maxwell's theory, but this vector has never been detected experimentally or otherwise. Yet its existence has been confirmed by the Bohm-Aharanov and similar experiments. As a vector it must have length and direction, yet it is located within individual geometrical points in three-dimensional space that have no size or direction. The simple solution is that the magnetic vector potential extends from a point in three-dimensional space into the fourth direction of space [40].

The same is true for DeBroglie's matter waves just as he foresaw [41] in 1927. The pilot wave merely spreads out in the fifth direction of space-time in conjunction with the normal matter wave that moves through three-dimensional space. At about the same time in history, William Wilson derived the Klein-Gordon equation in quantum theory [42] by assuming that the Schrödinger wave function $\Psi$ was a five-dimensional volume. So when a fivedimensional geometry is used, these last two ideas become the same problem. David Bohm's model of a quantum potential field [43] as well as his concept of the explicit and implicate [44], both of which are related to DeBroglie's pilot wave theory, can also be correlated to the SoFT model. The results of these research investigations and many more problematical aspects of both classical and modern physics can be easily explained within the SoFT model.

For example, quarks are not real particles. A simple application of special relativity electromagnetic waves $y$ and the quantum in combination with one another easily offers an 
alternate solution to the detected three-sidedness experienced by particles and nuclei during high energy collisions without invoking the concept of quark-particles. In a proton-proton collision, the incoming proton is Lorentz-Fitzgerald contracted relative to the target proton according to their relative speed. Once the incoming proton has contracted down to the quantum limit, equivalent to the 'effective width' of the 'sheet', any energy transferred in the collision will be passed on to the target proton in such a manner that the target proton's internal electrical stress is evenly split along (quantized) the particle's three constituent dimensions.

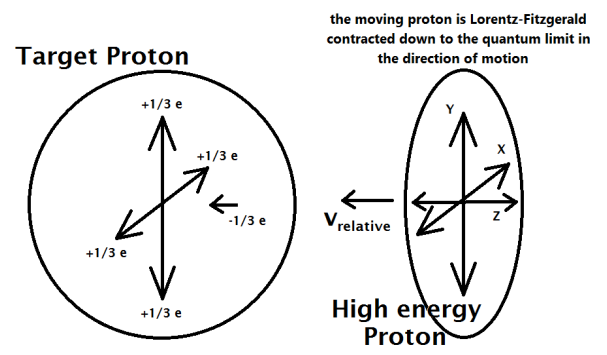

The value of $-1 / 3$ e-stress (internal electrical-stress) would occur along the direction or dimension of the moving proton or particle. It is pointed inward and thus negatively directed because it is a compression component due to the collision. The $+2 / 3$ (or $+1 / 3$ $+1 / 3$ ) e-stress components are thus pointed orthogonally outward along the other two dimensions of three-dimensional extended space. This must be true because the total internal stress can neither exceed nor be less than the original pre-collision estress of $+e$ since electronic charge must be conserved throughout the physical process of collision.

Electric charge is an internal electrical stress that creates an external strain in three-dimensional space and thus within the three-dimensionally extended 'sheet'. The initial e-stress has a different nature than the $g$-stresses that cause the force of gravity within three-dimensional space because the gstress of gravity is a surface tension effect along the top of the primary 'sheet'. The fact that the internal electrical stress breaks down into ratios of $n / 3$ is simply due to the fact that material space has three dimensions, which should render this particular explanation all the more obvious. A quark is just a descriptive term describing how that internal stress is quantized and redistributed along the three dimensions of space within a particle - according to a quantized Pascal's Principle - during a high energy collision or interaction with another real extended particle. So quarks are not particles in themselves, but rather the differently apportioned internal dimensions of space.

\section{All advances point to one conclusion}

It is actually easy to demonstrate how other quantum models reduce or merge into the single field model. In other words, once the 'point' of compatibility is realized the historical picture looks as if all other theories are directed toward a single field model.

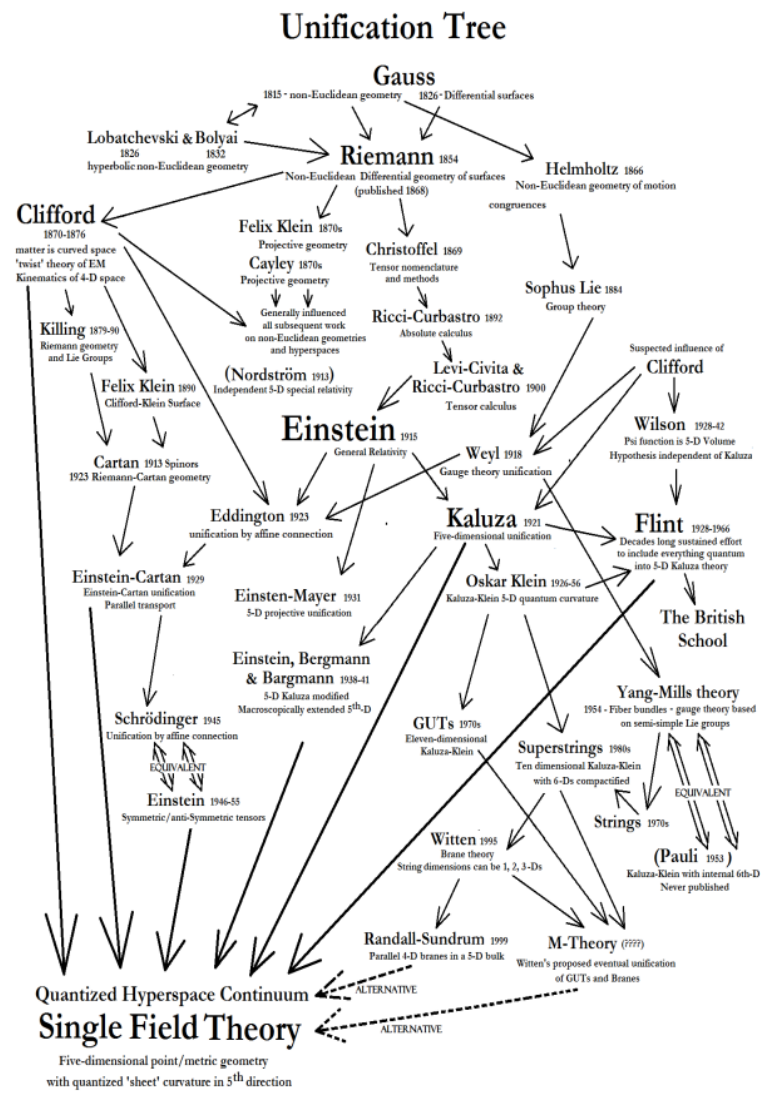

If all such theories are not advancing toward a single theory which is portrayed herein as the SoFT model of physical reality, then they are still progressing toward something like it. This fact must be true since nature is a single undivided thing, although it is presently misinterpreted through the "phallacies of modern fysics' as a duality of incomplete things or incomplete interpretations of reality.

The SoFT (Single operational Field Theory) model is directly related to the superstring model since both are derived from Kaluza's original fivedimensional model and both utilize Klein's implied notion that quantizing the fifth direction will quantize everything in the four-dimensional space-time continuum. If the original superstring model with Calabi-Yau bundles at each point in four-dimensional space-time were modified by placing a second fourdimensional surface (or brane) on top of the CalabiYau bundles to form a sandwich-like 'sheet' structure, a similar structure to teh SoFT 'sheet' would be formed.. 


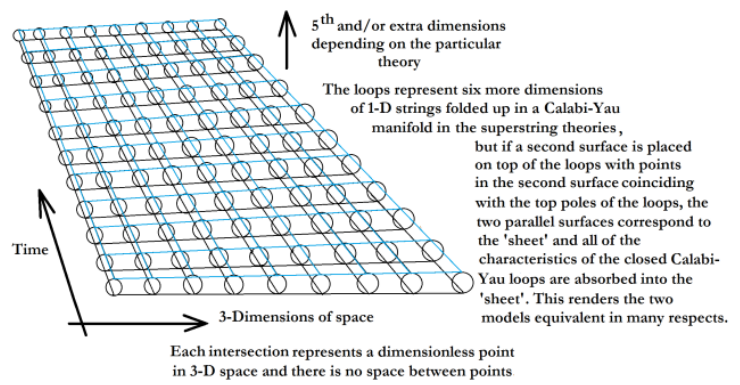

In this case, the physical properties associated with the six one-dimensional vibrating strings in the bundle would be absorbed or incorporated into the 'effective width' of the sandwich-like 'sheet'. In other words, the superstring model reduces to the SoFT model. The six dimensions of the Calabi-Yau bundle in the superstring theory are simply replaced by the 'effective width' of the $n=1$ 'sheet' in the SoFT model. This amounts to a membrane or 'brane' not unlike (but physically superior to) others proposed by modern theorists with an 'effective width' in the higher embedding dimension. One such example exists in the Randall-Sundrum Braneworld model.

The Braneworld model is certainly not new in physics. A similar model was suggested by Simon Newcomb in 1889 [45] to explain the null effects of the Michelson-Morley experiment. Furthermore, a related geometrical method could be applied to the Randall-Sundrum Braneworld to collapse it into the SoFT model. The secondary brane could be rotated through $180^{\circ}$ and moved to a position along the bottom of the primary four-dimensional brane. This geometrical maneuver would enclose the intervening five-dimensional bulk within the extra-dimensional extensions of individual discrete points on the branes.

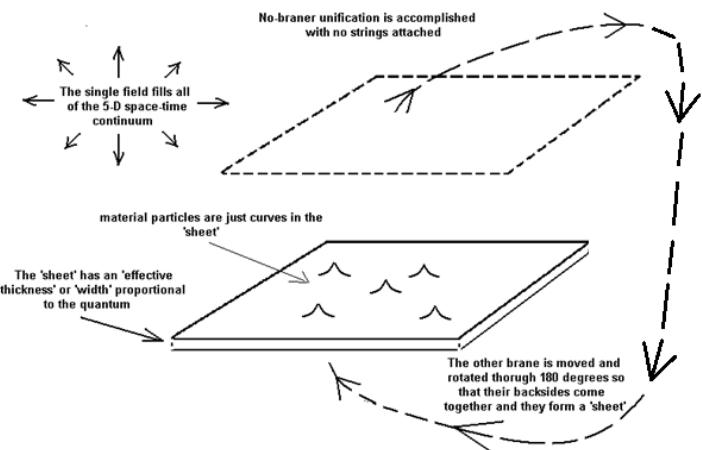

While the resulting 'sandwiched' double brane (primary and secondary branes are glued together) would form the 'sheet' characterized by its 'effective width', the original mathematical conditions upon which Kaluza's model was based - closure and equal length - would be restored to the Randall-Sundrum model. This would seem to be necessary since all such theories suffer from the same defect - how to deal with individual discrete dimensionless points within a continuum - that the SoFT model solves. It would thus appear that all present physical models reduce in the end or are at least progressing toward the SoFT or a SoFT-like model of material reality.

\section{Quantum and point}

A new philosophical interpretation of the quantum also favors this model. In the end, every quantum event must be rendered (after 'collapse' as some might say) relative to the space-time continuum in which other events share their common reality and outcomes. The method of this sharing, which 'collapses' the wave packet, is commonly called measurement. In a mental sense, the initial event (before 'collapse') would be 'non-local' (or undefined except by probabilities) anywhere in space and time with any possible momentum and energy. The act of measurement, previously called 'collapsing the wave function', does no more than 'localize' or lock the specified event into a well established position in space-time that can be represented by a space-time diagram.

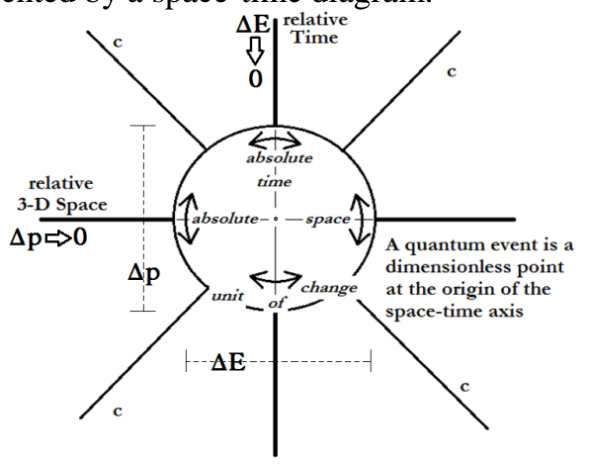

However, the uncertainties can never go to zero (or infinity) because the real physical localization process can never be more precise than a localized particle's extension in space which displaces an equal volume of space at a specific moment in time. This four-dimensional volume would be called the fundamental 'unit of change' of a quantized event.

Localizing the position of an interaction in space and time defines a specific 'unit of change' whose center point corresponds to the zero point or origin of a space-time diagram. Basically this means that the interaction can be represented by an energetic field resonance pattern that may or may not fulfill the geometrical and quantum properties of a real particle when it 'collapses' by aligning with the externally determined space-time diagram of the measurement agent (entanglement or consciousness). The space axis of the diagram corresponds to the infinite possibilities of uncertainty in momentum (the whole length of the spatial axes) when the uncertainty of position goes to zero (the origin). The same 
relationship occurs simultaneously between the time axis and the uncertainties in time and energy.

When this occurs, Planck's constant disappears as a factor (it is suppressed or hidden within the combined or connected space-time framework) when the event is localized in this manner and becomes 'real' in a quantum mechanical sense since $\Delta \mathrm{x} \Delta \mathrm{p}=\Delta \mathrm{E} \Delta \mathrm{t}$ at the origin of the space-time axes. The quantity of $h / 4 \pi$ is thus the connectivity or binding constant of space and time necessary to create spacetime while all of the uncertainty is bound up within the sphere that represents the shrinking measurement volume of the 'unit of change'. The act of measurement thus 'collapses' the uncertain quantum enigma (an amorphous energetic field resonance pattern) into the classical and relativistic reality of Newton, Einstein and Maxwell. Under these circumstances, the fundamental 'unit of change' represents a non-zero realization of the act of measurement.

Each fundamental 'unit of change' must have some small but finite non-zero constant value relative to the geometrical points in free-space by definition, more-or-less the smallest possible resonance pattern density in the single field as determined by field constants.

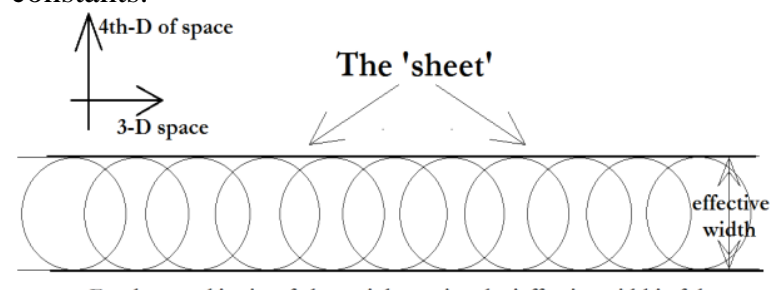

Fundamental 'units of change' determine the 'effective width' of the 'sheet' when they reach the lower limit of 'quantum measurement' The minimum unit of change must have a constant value (fixed volume) over free space if it is to have any meaning and significance in physics. It is not difficult to determine this value since it must also be related to the e-strain in free space. The e-strain in three-dimensional space yields a classical value known as the Coulomb force or $F=\left(1 / 4 \pi \varepsilon_{0}\right)\left(\mathrm{e}^{2} / \mathrm{r}^{2}\right)$ when two charged particles interact, but the distance factor of $r^{2}$ would disappear (go to infinity) in the case of a close approach to a particle's surface, just that point where the fundamental quantum 'unit of change' is reached or measured.

So the factor of $\mathrm{r}^{2}$ must be replaced by the limiting factors of space and time in the space-time diagram or $(4 \pi / \mathrm{hc})$, yielding a value of $\left(\mathrm{e}^{2} / 4 \pi \varepsilon_{0}\right)(4 \pi / \mathrm{hc})^{-1}$ which reduces to the fine structure constant. This value has no units, but still has to be related to the fundamental charged particles that create the e-strain in free space. Since particles are spherical and the e-strain spreads out spherically in space, the fine structure constant must be multiplied by the radius of a proton, $a_{0}$, to yield the proper value for the 'effective width' of the fundamental units of change, yielding approximately $\mathrm{a}_{0} / 137$. Then, in one last step, this value must be placed in terms of the higher fifth dimension. To do so requires that the fine structure constant be placed in terms of the magnetic potential vector which points in the fourth direction of space, as characterized by magnetic permeability $\mu_{0}$, rather than the electrical permittivity $\varepsilon_{0}$ which characterizes the scalar electric field in three-space. This yields an equivalent value of $\mathrm{e}^{2} \mathrm{c} \mu_{0} / 2 \mathrm{~h}$ as the true 'effective width' of the three-dimensional 'sheet' in the fourth direction of space.

\section{The tensor model}

The final mathematical model follows quite simply. The new term found in the classical gravity formula that represents the geometry of point-space yields a new anti- or non-symmetric term in the Einstein tensor. This term is associated with the definition of inertial mass, Mach's principle, a new centrifugal gravity component, Dark Matter and Dark Energy.

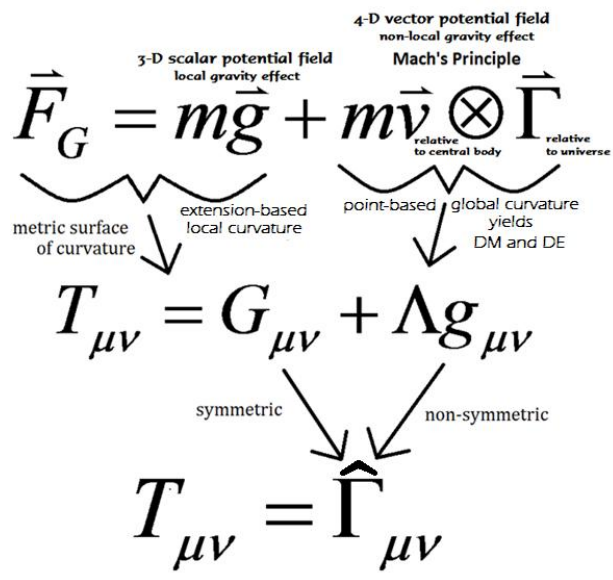

This new term is already known as Lambda-CDM in modern astrophysics and cosmology. However it is really so much more than just Lambda-CDM. Not only does this model complete the picture for general relativity, but it means that normal baryonic matter becomes the source Dark Matter in the universe.

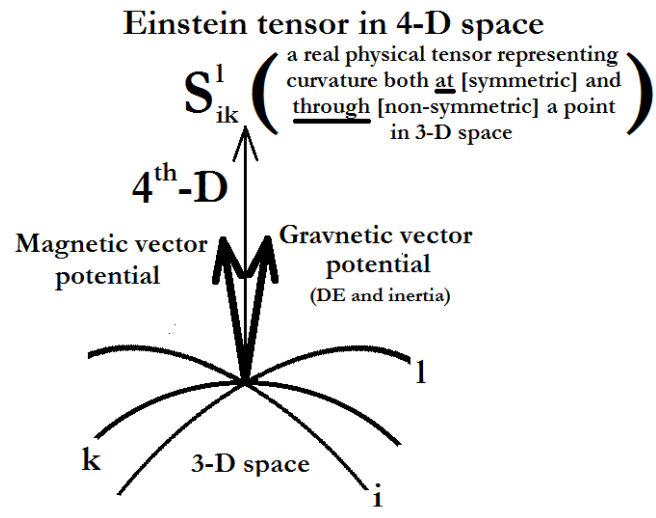


Dark Energy is essentially the gravnetic potential (not an energy as much as a pre-energy that interacts with baryonic matter) in free space that makes up most of the universe.

Since this new model of physical and material reality posits a five-dimensional single field characterized by electromagnetism and gravity as well as the five-dimensional properties of density variation and quantization, a higher-dimensional embedding sixth dimension is implied. The sixdimensional manifold would be represented by a single tensor $\mathbf{S}$ which splits into the electromagnetic and gravitational tensors in five-dimensional space. Then these two five-dimensional tensors split into two symmetric/non-symmetric tensors in fourdimensional space-time that represent the electric, magnetic, gravity and gravnetic fields in our commonly experienced space. The tensor $\mathbf{S}$ and the sixth dimension could be represented by either an infinite flat Euclidean space-time or an infinite dimensionless void. The sixth dimension represents a pre-spacetime because it would exist prior to space and time, which only become physical realities when the tensor splits into the five-dimensional space-time occupied by the single field. The notion that the fivedimensional space-time is embedded in a higher sixth flat dimension is not new, but was first presented by Edward Kasner in 1921 [46], shortly before Kaluza's original paper was published.

\section{Single Field Theory}

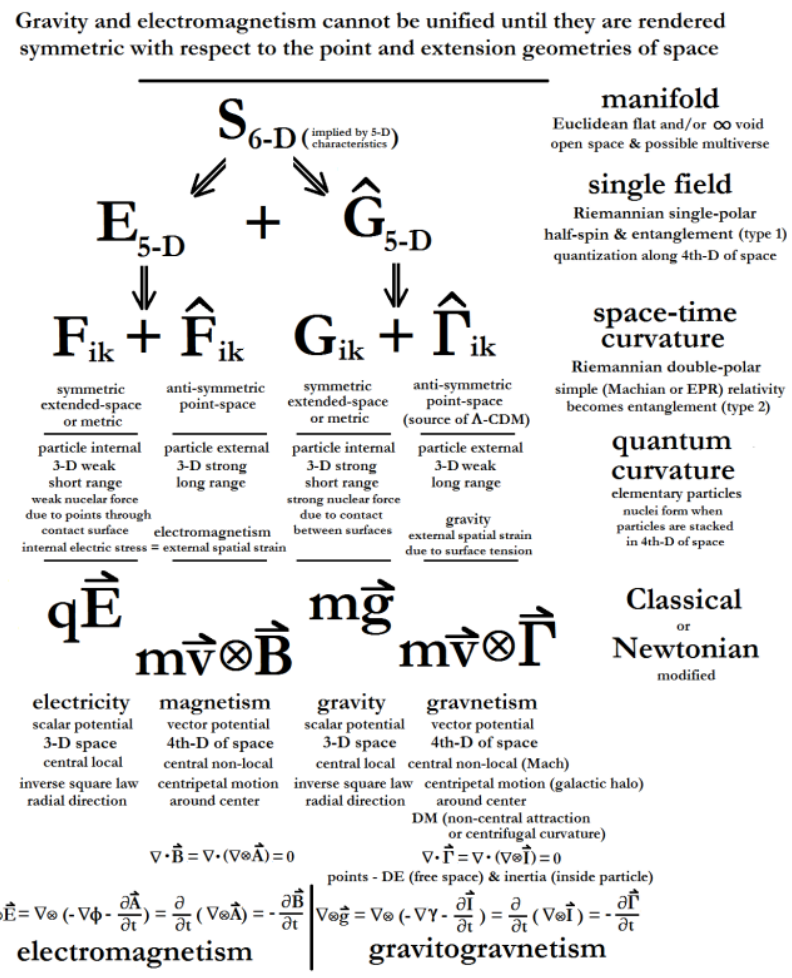

In either case, it is quite clear that the present paradigms of physics are vastly limited models that in some cases rise are rapidly approaching if not already reached the level of dead-end 'phallacies' that stand in the way of further progress and unification. These 'phallacies' are already inhibiting further theoretical research with their own domain of application in physics and will continue to do so as long as they are perceived as portraying reality without question. They are at least in need of completion to account for both the point-space and extension-space geometries that characterize physical reality if not a complete overhaul to render them compatible and capable of unification.

\section{References}

1. Gerhard Hessenberg. (1917) "Vectorielle Begründung der Differentialgeometrie," Math. Annalen 77:187-216.

2. Tullio Levi-Civita. (1917) "Nozione di parallelismo in una varieta qualunque e consequente specificione geometrica della curvatura riemanniana." Rend. Circ. Mat di Palermo 42: 173-215. Reprinted in Opere Mathamatische, Vol.4 (1917-1928). Bologna: A Coura Dell' Academia Nazionale dei Lincei, 1960: 1- 39.

3. Hermann Weyl. (1917) "Reine infinitesimale Geometrie." Math. Zeitschrift. Bd.2: 385-411; (1918) 201-216.

4. Hermann Weyl. (1918) "Gravitation und Electrizität." Sitz. Ber. Preuss. Akad. Wiss. 26: 465-480. Translated as "Gravitation and Electricity." In Einstein, et al. The Principles of Relativity. New York: Dover, 1952: Raum, Zeit, Materie. Translated by H.L. Brose as Space, Time, Matter. London: Methuen, 1922; New York: Dover, 1952; (1919) "Eine neue Erweitung der Relativitätstheorie." Ann. d. Phys. 59: 101.

5. Arthur Eddington. (1921) "A Generalisation of Weyl's Theory of the Electromagnetic Gravitational Fields." Proceedings of the Royal Society of London A99: 104-122.

6. Élie Cartan (1923) "Sur une généralisation de la notion de courbure de Riemann et les espaces à torsion." C. R. Acad. Sci. (Paris) 174 : 593-595.

7. Élie Cartan. (1923) "Sur les variétés à connexion affine et la théorie de la relativité généralisée." Part I: Ann. Éc. Norm. 40: 325-412, and (1924) 41: 1-25; Part II: (1925) 42: 17-88; La geometrie des espaces de Riemanns, fasc. 9 of Memorial des Sciences Mathematiques. Paris: 1925 ; 
"Espaces a connexion affine, projective et conforme." Acta Mathematica 48 (1926): 142 ; Lecons sur les geometrie des espaces Riemann, (Paris: 1928).

8. Albert Einstein (1929) "Zur Einheitlichen Feldtheorie." Sitz. Ber. Preuss. Akad. Wiss. 1: 2-7; "Field Theories Old and New." New York Times, 3 February, 1929. Reprinted New York; Readex Microprint, n.d.; "The New Field theory I, II." Observatory 51 (1929); (1930) "Auf die Riemann-Metric und den Fernparallelismus gegründte einheitliche Feldtheorie." Mathematische Annalen 102: 685-697.

9. Yu.V. Nachalov. (2003) "The Basics of Torsion Mechanics", 2003. Available online. Accessed 3 September 2007 at www.amasci.com/freenrg/tors/tors24.html; and E.A. Parkhomov. (2003) "Experimental detection of the torsion field." Available online. Accessed 29 September 2007 at www.amasci.com/freenrg/tors/doc15.html;

A.E. Akimov, A.E. and G.I. Shipov. "Torsion Fields and Their Experimental Manifestations." Proceedings of International Conference: New Ideas in Natural Science (1995). First page and bibliography reprinted: Available online. Accessed 29 August 2002 at www.eskimo.com/ billb/freenrg/tors/torsd.h tml>; also available as Preprint No 4a. Russian Academy of Natural Sciences, International Institute for Theoretical and Applied Physics, Moscow, 1995. Available online. Accessed 29 August 2002 at www.binet.lv/firms/project/english/torsion.h tm.

10. Wolfgang Pauli. (1921) Theory of Relativity, translated by G. Field. New York: Dover, 1981. Originally "Relativitätstheorie," Encyklopädie de mathematischen Wissenschafter 19. Leipzig: B.G. Teubner, 1921.

11. Theodor Kaluza. (1921) "Zum Unitätsproblem der Physik." Sitzungsberichte der Preussische Akademie der Wissenschaften 54: 966-972.

12. Oscar Klein. (1926) "Quantentheorie und Fünfdimensionale Relativitätstheorie." Zeitschrift fur Physik: 895-906; also "The Atomicity of Electricity as a Quantum Theory Law." Nature, 118: 516; (1927) “Zur Fünfdimensionale Darstellung der Relativitätstheorie." Zeitschrift fur Physik 46: 188-208.
13. Oscar Klein. (1939) "On the Theory of Charged Particles." New Theories in Physics, International Institute of Intellectual Cooperation, Paris; (1947) "Meson Fields and Nuclear Interaction." Arkiv for Mathematik, Astronomi och Fysik 34A: 1-19.

14. Albert Einstein. (1925) "Einheitliche theorie von Gravitation und Elektrizität." Sitzungsberichte der Preussischen Akademie der Wissenschaften (Berlin), Physickalischemathematische klasse: 414-419.

15. Frank R. Saxby. (1945) "Unification of the Physical Fields." Nature 151: 609-10.

16. Erwin Schrödinger. (1944) "The Affine Connexion in Physical Field Theory." Nature 53: 572-575.

17. Albert Einstein. (1945) "A Generalization of the Relativity Theory of Gravitation." Annals of Mathematics 46: 578-84; and E.G. Strauss (1946) "A Generalization of the Relativistic Theory of Gravitation II." Annals of Mathematics 47: 731- 741.

18. Albert Einstein. (1956) The Meaning of Relativity, sixth edition. Princeton: Princeton University Press.

19. C. Peter Johnson. (1953) "A Criticism of a Recent Unified Field Theory," Letters to the Editor. Physical Review 89: 320-1; Einstein "A Comment on a Criticism of Unified Field Theory," Letters to the Editor. Physical Review 89: 321; Einstein and B. Kaufman (1955) "A New Form of the General Relativistic Field Equations." Annals of Mathematics 62: 128-18.

20. John W. Moffat. (1979) "New theory of gravitation." Phys. Rev. D 19: 3554-3558; (1995) "Nonsymmetric Gravitational Theory." Phys. Lett. B 355: 447452. Available online at arXiv:grqc/9411006.

21. Tilman Sauer. (2007) "Einstein's Unified Field Theory Program." Einstein Papers Project. Available online at philsciarchive.pitt.edu/3293/1/uft.pdf; "Field equations in teleparallel spacetime: Einstein's Fernparallelismus approach towards unified field theory." Available online at arxiv.org/pdf/physics/0405142.pdf.

22. Hubert F.M. Gönner. (2004) "On the History of Unified Field Theories." Living Reviews in Relativity 7, 2; Erhard Scholz. "Curved spaces: Mathematics and Empirical evidence, ca. 1830-1923." 2005. Preprint at Wuppertal and mathg.uniwuppertal.de/ scholz/preprints/ES_OW200 
5.pdf. Shorter version to appear at Oberwalfach Reports; Erhard Scholz. "H.Weyl's and E. Cartan's proposal for infinitesimal geometry in the early 1920s." Available online at www2.math.uniwuppertal.de/ scholz/preprints/weyl_cartan. pdf.

23. James Shifflett. (2005) A modification of Einstein-Schrodinger theory that contains both general relativity and electrodynamics. $\mathrm{PhD}$ dissertation. Available online at arxiv.org/pdf/0801.2307v2.pdf.

24. Albert Einstein and Peter G. Bergmann. (1938) "On a Generalization of Kaluza's Theory of Electricity." Annals of Mathematics 39: 683-701; Albert Einstein, Peter Bergmann and Valentin Bargmann. (1941) "On the Five Dimensional Representation of Gravitation and Electricity." In the Theodor von Karman Anniversary Volume. Pasadena, 1941: 212225.

25. Lisa Randall and Raman Sundrum. (1999) "A Large Mass Hierarchy from a Small Extra Dimension." Physical Review Letters 83: 3370-3373. Available online at ArXiv: hep-ph/9905221; (1999) "An alternative to compactification." Physical Review Letters 83: 4690-4693. Available online at ArXiv:hep-th/9906064.

26. David Hilbert. (1900) "Mathematical Problems." Lecture delivered before the International Congress of Mathematicians at Paris. Available online at aleph0.clarku.edu/ djoyce/hilbert/problems. html.

27. Hermann Weyl. (1927, 1949) Philosophy of Mathematics and Natural Science. (1949; an enlarged English version of a 1927 work Philosophie der Mathematik und Naturwissenschaften), and Symmetry (1952): 44.

28. James E Beichler. (2013) Phallacies in Fysics. Volume 1: The untold story of Classical Physics. Published and available online as a Kindle e-book.

29. Oliver Heaviside (1893) "A gravitational and electromagnetic analogy." The Electrician 31: 81-82.

30. James E. Beichler. (2013) Four Pillars of Wisdom: Fundamental concepts of natural science. Published and available online as a Kindle e-book.

31. James E. Beichler (2012) "The Tie that Binds: The fundamental unit of 'change' in space and time." In The Physics of Reality:
Space, Time, Matter, Cosmos Proceedings of the 8th Symposium Honoring Mathematical Physicist Jean-Pierre Vigier, edited by Richard L Amoroso, Louis H Kauffman and Peter Rowlands. World Scientific Publishing Company, September 18, 2013; James E. Beichler . (2013) "The Point of Unification in Theoretical Physics." In Proceedings of the 20th NPA Conference. Available online at www.worldsci.org/pdf/abstracts/abstracts_p aperlink_7066.docx.

32. William Kingdon Clifford. (1873) "Preliminary Sketch of Biquaternions." Proceedings of the London Mathematical Society: 381-395; Reprinted in Mathematical Papers, edited by Robert Tucker with a preface by H.J. Stephen Smith. Reproduction of 1882 original: New York: Chelsea Publishing, 1968; 181-200; Elements of Dynamic: An Introduction to the Study of Motion and Rest in Solid and Fluid Bodies, Part I: Kinematics. London: Macmillan, 1878; James E. Beichler. (2013) Twist and Shout: Clifford's (not so) secret program for 'Solving the Universe'. Published and available online as an Amazon Kindle e-book.

33. Einstein. Meaning of Relativity: 93-94.

34. James E. Beichler. (2014) "Consciousness of Unification: The Mind-Matter phallacy bites the dust." Proceedings of the Vigier IX Conference, edited by Richard Amaroso.

35. James E. Beichler. (1980, 2013) Hyperspace Continuum: The five-dimensional continuum approach to a unified field theory. Available online as a Kindle e-book; Part 1, Hyperspace, was originally written as $A$ Five-Dimensional Continuum Approach to a Unified Field Theory. Master's Thesis at San Francisco State University, December 1980.

36. $\mathrm{Vu}$ B. Ho. (1995) "A geometric formulation of strong interaction." Available online at arxiv.org/abs/hep-th/9504072.

37. Henry T. Flint. (1945) "Quantum Equations and Nuclear Field Theories." Philosophical Magazine 36: 635-643.

38. Albert Einstein, Boris Podolsky and Nathan Rosen. (1935) "Can Quantum Mechanical Description of Reality be Considered Complete?" Physical Review 47: 777-780.

39. Edmund T. Whittaker (1903) "On the Partial Differential Equations of Mathematical Physics." Math. Ann. 57: 333-355; (1904) "On an Expression of the Electromagnetic 
Field Due to Electrons by Means of Two Scalar Potential Functions." Proc. Lond. Math. Soc. 1 : 367-372.

40. James E. Beichler. (2007) “Three Logical Proofs: The Five-Dimensional Reality of Space-Time." Journal of Scientific Exploration 21, 3: 523-542. Available online at scientificexploration.org/journal/jse_21_3_b eichler.pdf.

41. Louis DeBroglie. (1927) Solvay Conference. Published 1928, Electrons et Photons: Rapports et Descussions du Cinquieme Conseil de Physique tenu a Bruxelles du 24 au 29 October 1927 sous les auspices de l'Institut International Physique Solvay; (1987) "Interpretation of quantum mechanics by the double solution theory." Annales de la Louis de Broglie 12 : 1-23. Available online at aflb.ensmp.fr/AFLBclassiques/aflb124p001.pdf.

42. William Wilson. (1928) "Relativity and Wave Mechanics." Proceedings of the Royal Society 118: 441-448.

43. David Bohm and B.J. Hiley. (1975) On the intuitive understanding of nonlocality as implied by quantum theory. Foundations of Physics, Volume 5, Number 1: 93-109; and P. N. Kaloyerou. (1987) "An ontological basis for the quantum theory." Physics Reports (Review section of Physics Letters) 144: 321-375.

44. David Bohm. (1990) "A new theory of the relationship of mind and matter." Philosophical Psychology 3 (2): 271-286; Bohm and B.J. Hiley. (1993). The Undivided Universe: An ontological interpretation of quantum theory. London: Routledge.

45. Simon Newcomb (1889) "On the Fundamental Concepts of Physics." Abstract in the Bulletin of the Washington Philosophical Society, 11 (1888/1891): 514515; A roughly typed copy of the complete talk can be found in Box \#94, Newcomb Papers, Library of Congress.

46. Edward Kasner. (1921) "The Einstein Solar Field and Space of Six Dimensions." Science 53: 238-239. 